\title{
ON THE CONVERGENCE OF THE FOURIER APPROXIMATION FOR EIGENVALUES AND EIGENFUNCTIONS OF DISCONTINUOUS PROBLEMS*
}

\author{
M. S. MIN ${ }^{\dagger}$ AND D. GOTTLIEB ${ }^{\dagger}$
}

\begin{abstract}
In this paper, we consider a model eigenvalue problem with discontinuous coefficients in order to study the convergence of the Fourier methods applied to this problem. We prove that the rate of convergence of the Fourier-Galerkin method is third order for the eigenvalues and order 2.5 for the eigenfunctions. For the Fourier collocation method we obtained only second order accuracy.

We also show that the Fourier collocation method can be improved by a preprocessing of the coefficients.
\end{abstract}

The theory is confirmed by numerical results.

Key words. discontinuous problems, Fourier-Galerkin method, Fourier collocation method, minmax principle

AMS subject classifications. $65 \mathrm{~N} 15,65 \mathrm{~N} 25,65 \mathrm{~N} 35$

PII. S0036142902403012

1. Introduction. The paper is motivated by an issue arising in the use of spectral methods in nonlinear optics. The Fourier methods when applied to problems in nonlinear optics are extremely fast, and if the problem is smooth they provide high order accuracy. However, when different media are considered, the coefficients are only piecewise smooth and the accuracy is lost.

In order to understand the phenomenon, and as a first step to improve the accuracy of the Fourier schemes in those circumstances, we consider in this paper a model eigenvalue problem with piecewise constant coefficients and study the convergence of the Fourier-Galerkin and Fourier collocation methods to the eigenvalues and the eigenfunctions of this problem. The surprising fact is that the order of convergence of the eigenvalues obtained by the Fourier-Galerkin method is cubic. When the Fourier collocation method is applied, the results are only second order. Those results are proven and supported by numerical computations.

It turns out that, by preprocessing the discontinuous coefficients, one can improve the accuracy of the collocation method. In fact, if one uses the point values of the finite Fourier series of the coefficients instead of the point values of the coefficients themselves, one recovers third order accuracy for the eigenvalues and order 2.5 for the eigenfunction.

The paper is organized as follows. In section 2, we present the problem and show some of the eigenvalues and eigenfunctions. In section 3 , we rewrite the problem in its variational form and quote some relevant facts. In section 4, we discuss the FourierGalerkin method and prove the order of accuracy. Section 5 is devoted to the Fourier collocation method and the error estimates of this method. In section 6 , we show how to improve the accuracy of the collocation method.

* Received by the editors February 21, 2002; accepted for publication (in revised form) June 24, 2002; published electronically January 7, 2003. This work was supported by AFOSR grant F4962099-1-0077, DOE grant DE-FG02-98ER25346, and NSF grant DMS-9804985.

http://www.siam.org/journals/sinum/40-6/40301.html

${ }^{\dagger}$ Division of Applied Mathematics, Brown University, Providence, RI 02912 (msmin@cfm.brown. edu,dig@cfm.brown.edu). 
We regard this paper as the first step toward recovering exponential accuracy for this problem.

2. The discontinuous eigenvalue problem. Consider the following eigenvalue problem with a piecewise constant coefficient:

$$
-\frac{d^{2} u}{d x^{2}}=\lambda \epsilon(x) u \quad \text { for } \quad x \in(-\pi, \pi),
$$

where $\epsilon(x)=1$ in $(-\pi, 0)$ and $\epsilon(x)=\beta^{2}$ in $[0, \pi), \beta \neq 1$. The $H_{p}^{2}[-\pi, \pi]$ eigenfunction $u_{l}(x)$ (the $p$ stands for periodic) is given by

$$
u_{l}(x)=\left\{\begin{array}{rr}
C \cos \left(\sqrt{\lambda}_{l} x\right)+\beta D \sin \left(\sqrt{\lambda}_{l} x\right), & -\pi \leq x \leq 0 \\
C \cos \left(\beta \sqrt{\lambda}_{l} x\right)+D \sin \left(\beta \sqrt{\lambda_{l}} x\right), & 0 \leq x \leq \pi
\end{array}\right.
$$

where the constants $C, D$ and the eigenvalue $\lambda_{l}$ are determined by the demand that the system

$$
\begin{array}{r}
C(\cos \sqrt{\lambda} \pi-\cos \beta \sqrt{\lambda} \pi)+D(-\beta \sin \sqrt{\lambda} \pi-\sin \beta \sqrt{\lambda} \pi)=0, \\
C(\sin \sqrt{\lambda} \pi+\beta \sin \beta \sqrt{\lambda} \pi)+D(\beta \cos \sqrt{\lambda} \pi-\beta \cos \beta \sqrt{\lambda} \pi)=0
\end{array}
$$

has a nontrivial solution. Considering the case $\beta=2$, for $y=\cos \sqrt{\lambda} \pi$, the eigenvalues $\lambda$ satisfy the equation

$$
(y-1)\left(9 y^{2}+9 y+2\right)=0,
$$

and so there are families of eigenvalues determined by

$$
\cos \sqrt{\lambda} \pi=1,-\frac{1}{3}, \quad \text { or }-\frac{2}{3} .
$$

The first five analytic eigenvalues (with six digits of precision) and the corresponding eigenvectors are shown in Figure 1. For comparison, we also carry the same procedure for $\beta=3$, where the analytic eigenvalues are determined by

$$
\cos \sqrt{\lambda} \pi= \pm 1 \text { or } \pm \frac{1}{4} .
$$

In this paper, we examine the rate of convergence of the Fourier methods (Galerkin and collocation) as a first step in an effort to improve the rate of convergence and be able to also apply the Fourier methods for this discontinuous problem.

3. The variational formulation. We define two inner products:

$$
\begin{aligned}
& a(u, v)=\int_{-\pi}^{\pi} u^{\prime}(x) \overline{v^{\prime}(x)} d x, \\
& (u, v)=\int_{-\pi}^{\pi} u(x) \overline{v(x)} \epsilon(x) d x .
\end{aligned}
$$

Following Strang and Fix [7, p. 220], the eigenvalue problem (2.1) can be presented in the following variational form: finding a scalar $\lambda$ and a function $u \in H_{p}^{1}[-\pi, \pi]$ such that

$$
a(u, v)=\lambda(u, v)
$$




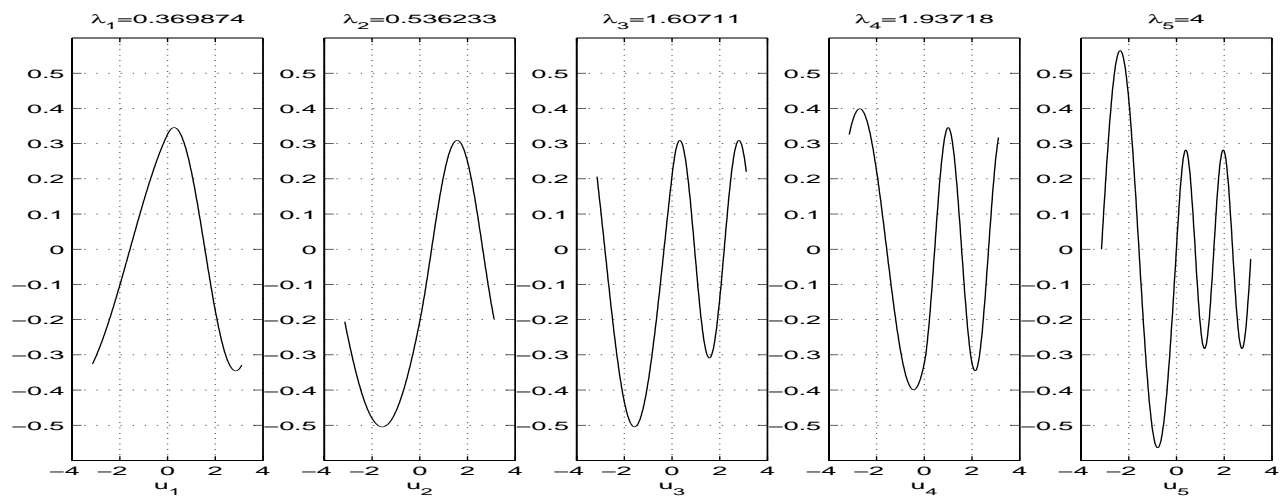

FIG. 1. The first five analytic eigenvalues and eigenfunctions.

for all $v$ in the Hilbert space $H_{p}^{1}[-\pi, \pi]$. Note that $a(u, v)$ is Hermitian.

Our proofs will use extensively the minmax principle [3].

TheOREm 3.1. Let $\lambda_{l}$ denote the eigenvalues of (2.1) and $S_{l}$ be any l-dimensional subspace of $H_{p}^{1}[-\pi, \pi]$. Then, for $\lambda_{1} \leq \lambda_{2} \leq \cdots \leq \lambda_{l} \cdots$,

$$
\lambda_{l}=\min _{S_{l} \subset H_{p}^{1}[-\pi, \pi]} \max _{v \in S_{l}} \frac{a(v, v)}{(v, v)} .
$$

In this paper, we will use sharper characterizations of the eigenvalues.

LEMma 3.2. Let $\lambda_{i}$ be arranged in an ascending order and define

$$
E_{i, j}=\operatorname{span}\left\{u_{i}, \ldots, u_{j}\right\}
$$

where $u_{i}$ is the eigenfunction corresponding to the eigenvalue $\lambda_{i}$. Then

$$
\begin{aligned}
& \lambda_{l}=\max _{v \in E_{k, l}} \frac{a(v, v)}{(v, v)}, \quad k \leq l, \\
& \lambda_{l}=\min _{v \in E_{l, m}} \frac{a(v, v)}{(v, v)}, \quad l \leq m .
\end{aligned}
$$

4. Fourier-Galerkin method. It is natural to consider the Fourier method to approximate the periodic problem. Here, we introduce the Fourier-Galerkin method applied to the variational formulation for approximating the eigenvalues and eigenfunctions.

Let $\mathcal{P}_{\mathcal{N}}$ be the space of the trigonometric polynomials of degree $N / 2$ defined as

$$
\mathcal{P}_{\mathcal{N}}=\operatorname{span}\left\{e^{i k x} \mid-N / 2 \leq k \leq N / 2\right\} .
$$

In this subspace, we look for $\lambda^{N}$ and $u^{N}$ such that

$$
a\left(u^{N}, v^{N}\right)=\lambda^{N}\left(u^{N}, v^{N}\right) \text { for all } v^{N} \in \mathcal{P}_{\mathcal{N}} ;
$$

in other words,

$$
\int_{-\pi}^{\pi}\left(u^{N}(x)\right)^{\prime} \overline{\left(v^{N}(x)\right)^{\prime}} d x=\lambda^{N} \int_{-\pi}^{\pi} u^{N}(x) \overline{v^{N}(x)} \epsilon(x) d x .
$$


4.1. Numerical scheme and its results. The approximate eigenfunction $u^{N}$ in the subspace $\mathcal{P}_{\mathcal{N}}$ is expanded by

$$
u^{N}=\sum_{k=-\frac{N}{2}}^{\frac{N}{2}}\left(\hat{u}^{N}\right)_{k} e^{i k x} .
$$

Substituting $u^{N}$ into the variational formulation (4.2) with $v^{N}=e^{i n x}$, and denoting the vector of the coefficients by $\hat{\mathbf{u}}^{N}$, we get a generalized eigenvalue problem in a matrix form as

$$
K \hat{\mathbf{u}}^{N}=\lambda^{N} M \hat{\mathbf{u}}^{N},
$$

where

$$
K_{n k}=\int_{-\pi}^{\pi}(k \cdot n) e^{i(k-n) x} d x \text { and } \quad M_{n k}=\int_{-\pi}^{\pi} e^{i(k-n) x} \epsilon(x) d x .
$$

Solving the matrix eigenvalue problem (4.5) computationally using a proper eigensolver, we obtain the approximate $l$ th eigenvalues, $\lambda_{l}^{N}(l \leq N)$, and the set of orthogonal vectors $\hat{\mathbf{u}}_{l}^{N}=\left[\left(\hat{u}_{l}^{N}\right)_{-N / 2}, \ldots,\left(\hat{u}_{l}^{N}\right)_{N / 2}\right]^{T}$ which is used to approximate the $l$ th eigenfunction $u_{l}$ as a finite Fourier series $u_{l}^{N}$. In Tables 1 and 2, the orders of the relative errors for $\lambda_{l}^{N}-\lambda_{l}$ and the discrete $L_{2}$-errors of $u_{l}-u_{l}^{N}$ are provided for the first five eigenvalues in ascending order and for the associated eigenfunctions. We note the surprising fact that the Galerkin approximation to the eigenvalue problem (2.1) converges with third order accuracy for the eigenvalues and order 2.5 for the eigenfunctions even though the eigenfunctions are only in $H_{p}^{2}$. In fact, we will show in Lemma 4.5 that the eigenfunctions are in $H_{p}^{\frac{5}{2}-\epsilon}$ for any $\epsilon>0$.

4.2. Error estimates for eigenvalues and eigenfunctions. In this section, we provide the error estimates for the approximate eigenvalues and eigenfunctions for the Fourier-Galerkin method.

We first treat the approximate eigenvalues. Let $P_{N} u$ be the $\frac{N}{2}$ th order truncated Fourier series of $u$. (We will denote also $P=P_{N}$.) It is clear that it satisfies

$$
a\left(u-P_{N} u, v^{N}\right)=0 \text { for all } v^{N} \in \mathcal{P}_{\mathcal{N}} .
$$

It is true that the minmax principle is also valid for the Galerkin procedure:

$$
\lambda_{l}^{N}=\min _{S_{l} \subset \mathcal{P}_{\mathcal{N}}} \max _{v \in S_{l}} \frac{a(v, v)}{(v, v)} .
$$

Lemma 4.1. Let $\lambda_{l}^{N}$ be the approximation to $\lambda_{l}$ which is obtained by the Galerkin procedure. Then

$$
\lambda_{l} \leq \lambda_{l}^{N} \leq \lambda_{l} \max _{v \in E_{1, l}} \frac{(v, v)}{(P v, P v)}
$$

Proof. Due to the minmax principle (3.4) and (4.8), we have

$$
\begin{aligned}
\lambda_{l} & =\min _{S_{l} \subset H_{p}^{1}[-\pi, \pi]} \max _{v \in S_{l}} \frac{a(v, v)}{(v, v)} \\
& \leq \min _{S_{l} \subset \mathcal{P}_{\mathcal{N}}} \max _{v \in S_{l}} \frac{a(v, v)}{(v, v)}=\lambda_{l}^{N} .
\end{aligned}
$$


TABLE 1

The relative errors of eigenvalues for the case $\beta=2$ and the discrete $L_{2}$-errors of $u_{i}-u_{i}^{N}$ for the Fourier-Galerkin method.

\begin{tabular}{|c||c||c||c|c||c|c|}
\hline$\lambda_{i}$ & $N$ & $\lambda_{i}^{N}$ & $\frac{\left(\lambda_{i}^{N}-\lambda_{i}\right)}{\lambda_{i}}$ & Order & $\left\|u_{i}-u_{i}^{N}\right\|_{l_{2}}$ & Order \\
\hline \multirow{5}{*}{0.36987} & 16 & 0.36991 & $1.0269(-4)$ & & $4.1430(-4)$ & \\
& 32 & 0.36988 & $1.5247(-5)$ & 2.7517 & $8.9399(-5)$ & 2.2124 \\
& 64 & 0.36988 & $2.0853(-6)$ & 2.8702 & $1.7382(-5)$ & 2.3627 \\
& 128 & 0.36988 & $2.7295(-7)$ & 2.9335 & $3.2192(-6)$ & 2.4328 \\
& 256 & 0.36987 & $3.4960(-8)$ & 2.9649 & $5.8234(-7)$ & 2.4668 \\
\hline \multirow{5}{*}{0.53623} & 16 & 0.53628 & $8.6637(-5)$ & & $5.7658(-4)$ & \\
& 32 & 0.53624 & $1.0678(-5)$ & 3.0203 & $1.0045(-4)$ & 2.5210 \\
& 64 & 0.53623 & $1.3287(-6)$ & 3.0066 & $1.7617(-5)$ & 2.5114 \\
& 128 & 0.53623 & $1.6580(-7)$ & 3.0025 & $3.1020(-6)$ & 2.5057 \\
& 256 & 0.53623 & $2.0643(-8)$ & 3.0057 & $5.4732(-7)$ & 2.5028 \\
\hline \multirow{5}{*}{1.60712} & 16 & 1.60758 & $2.8694(-4)$ & & $1.8606(-3)$ & \\
& 32 & 1.60717 & $3.2907(-5)$ & 3.1243 & $3.0781(-4)$ & 2.5957 \\
& 64 & 1.60712 & $4.0121(-6)$ & 3.0360 & $5.3130(-5)$ & 2.5344 \\
& 128 & 1.60712 & $4.9821(-7)$ & 3.0095 & $9.3136(-6)$ & 2.5121 \\
& 256 & 1.60712 & $6.2253(-8)$ & 3.0005 & $1.6412(-6)$ & 2.5046 \\
\hline \multirow{5}{*}{1.93718} & 16 & 1.93833 & $5.9203(-4)$ & & $2.4795(-3)$ & \\
& 32 & 1.93734 & $8.1996(-5)$ & 2.8520 & $4.8917(-4)$ & 2.3416 \\
& 64 & 1.93720 & $1.0998(-5)$ & 2.8983 & $9.2481(-5)$ & 2.4031 \\
& 128 & 1.93718 & $1.4324(-6)$ & 2.9408 & $1.6966(-5)$ & 2.4465 \\
& 256 & 1.93718 & $1.8343(-7)$ & 2.9651 & $3.0582(-6)$ & 2.4719 \\
\hline \multirow{5}{*}{4.00000} & 16 & 4.00094 & $2.3385(-4)$ & & $3.4923(-3)$ & \\
& 32 & 4.00002 & $5.9398(-6)$ & 5.2990 & $2.6560(-4)$ & 3.7168 \\
& 64 & 4.00000 & $1.7675(-7)$ & 5.0706 & $2.2714(-5)$ & 3.5476 \\
& 128 & 4.00000 & $5.4572(-9)$ & 5.0174 & $1.9917(-6)$ & 3.5115 \\
& 256 & 4.00000 & $1.6993(-10)$ & 5.0051 & $1.7822(-7)$ & 3.4822 \\
\hline
\end{tabular}

TABle 2

The relative errors of eigenvalues for the case $\beta=3$ and the discrete $L_{2}$-errors of $u_{i}-u_{i}^{N}$ for the Fourier-Galerkin method.

\begin{tabular}{|c||c||c||c|c||c|c|}
\hline$\lambda_{i}$ & $N$ & $\lambda_{i}^{N}$ & $\frac{\left(\lambda_{i}^{N}-\lambda_{i}\right)}{\lambda_{i}}$ & Order & $\left\|u_{i}-u_{i}^{N}\right\|_{l_{2}}$ & Order \\
\hline \multirow{5}{*}{0.17603} & 16 & 0.17606 & $1.5651(-4)$ & & $8.6969(-4)$ & \\
& 32 & 0.17604 & $2.3223(-5)$ & 2.7526 & $1.7589(-4)$ & 2.3059 \\
& 64 & 0.17603 & $3.1759(-6)$ & 2.8703 & $3.2960(-5)$ & 2.4159 \\
& 128 & 0.17603 & $4.1565(-7)$ & 2.9337 & $5.9857(-6)$ & 2.4611 \\
& 256 & 0.17603 & $5.3176(-8)$ & 2.9665 & $1.0719(-6)$ & 2.4814 \\
\hline \multirow{5}{*}{0.33690} & 16 & 0.33704 & $4.3531(-4)$ & & $2.1237(-3)$ & \\
& 32 & 0.33691 & $5.3609(-5)$ & 3.0215 & $3.7423(-4)$ & 2.5046 \\
& 64 & 0.33690 & $6.6757(-6)$ & 3.0055 & $6.6210(-5)$ & 2.4988 \\
& 128 & 0.33690 & $8.3365(-7)$ & 3.0014 & $1.1722(-5)$ & 2.4978 \\
& 256 & 0.33690 & $1.0418(-7)$ & 3.0004 & $2.0744(-6)$ & 2.4984 \\
\hline \multirow{5}{*}{1.00000} & 16 & 1.00005 & $4.7257(-5)$ & & $2.1107(-3)$ & \\
& 32 & 1.00000 & $1.8636(-6)$ & 4.6643 & $3.6870(-4)$ & 2.5172 \\
& 64 & 1.00000 & $6.6628(-8)$ & 4.8059 & $6.5495(-5)$ & 2.4930 \\
& 128 & 1.00000 & $2.2386(-9)$ & 4.8955 & $1.1610(-5)$ & 2.4959 \\
& 256 & 1.00000 & $7.2292(-11)$ & 4.9526 & $2.0544(-6)$ & 2.4986 \\
\hline \multirow{5}{*}{2.01518} & 16 & 2.02204 & $3.4072(-3)$ & & $2.6579(-2)$ & \\
& 32 & 2.01587 & $3.4220(-4)$ & 3.3157 & $3.0104(-3)$ & 3.1423 \\
& 64 & 2.01526 & $4.0586(-5)$ & 3.0758 & $4.5219(-4)$ & 2.7349 \\
& 128 & 2.01519 & $5.0069(-6)$ & 3.0190 & $7.4652(-5)$ & 2.5987 \\
& 256 & 2.01518 & $6.2380(-7)$ & 3.0048 & $1.2792(-5)$ & 2.5449 \\
\hline \multirow{5}{*}{2.49776} & 16 & 2.50541 & $3.0620(-3)$ & & $1.0310(-2)$ & \\
& 32 & 2.49866 & $3.5823(-4)$ & 3.0955 & $2.1636(-3)$ & 2.2526 \\
& 64 & 2.49788 & $4.6049(-5)$ & 2.9596 & $4.2364(-4)$ & 2.3525 \\
& 128 & 2.49778 & $5.9303(-6)$ & 2.9570 & $8.0203(-5)$ & 2.4011 \\
& 256 & 2.49776 & $7.5555(-7)$ & 2.9725 & $1.4749(-5)$ & 2.4430 \\
\hline
\end{tabular}


Thus only the upper bound of the approximate eigenvalue is left to be investigated.

Let $P E_{1, l}$ be spanned by $P u_{1}, \ldots, P u_{l}$. Then it is clear that $P E_{1, l}$ is the $l$ dimensional subspace of $\mathcal{P}_{\mathcal{N}}$. Using the minmax principle (4.8),

$$
\lambda_{l}^{N} \leq \max _{v \in P E_{1, l}} \frac{a(v, v)}{(v, v)}=\max _{v \in E_{1, l}} \frac{a(P v, P v)}{(P v, P v)} .
$$

Note that in $[7]$ we have

$$
a(v, v)=a(P v, P v)+2 a(v-P v, P v)+a(v-P v, v-P v) .
$$

From (4.7), we know that $a(v-P v, P v)$ always vanishes for all $P v$ in the space $\mathcal{P}_{\mathcal{N}}$. Then we have $a(P v, P v) \leq a(v, v)$. Thus,

$$
\lambda_{l}^{N} \leq \max _{v \in E_{1, l}} \frac{a(v, v)}{(P v, P v)}=\max _{v \in E 1, l} \frac{a(v, v)}{(v, v)} \cdot \frac{(v, v)}{(P v, P v)} \leq \lambda_{l} \cdot \max _{v \in E_{1, l}} \frac{(v, v)}{(P v, P v)} .
$$

The last inequality is a by-product of (3.5). Thus the lemma is proven.

The issue is how close $\left(P_{N} v, P_{N} v\right)$ is to $(v, v)$ for $v \in E_{1, l}$. One would expect the second order accuracy in $N$ because of the smoothness of the eigenfunctions $u_{i}$. However, we will show that it is really third order. We start by examining the Fourier coefficients of the eigenfunctions.

LEMma 4.2. The Fourier coefficients $\left(\hat{u}_{l}\right)_{k}$ of the eigenfunction $u_{l}$ decay as $O\left(k^{-3}\right)$; in fact,

$$
\left(\hat{u}_{l}\right)_{k} \leq C k^{-3}\left\{\left|u_{l}(0)\right|+\frac{1}{k}\left|u_{l}^{\prime}(0)\right|+\frac{\lambda_{l}}{k}|| u_{l}||\right\},
$$

where $\left\|u_{l}\right\|$ is the $L_{2}$-norm of $u_{l}$.

Proof. Letting $u_{l}=u$ for simplicity, and using the fact that $u$ ' is continuous,

$$
\hat{u}_{k}=\frac{1}{2 \pi} \int_{-\pi}^{\pi} u e^{-i k x} d x=\frac{1}{2 \pi i k} \int_{-\pi}^{\pi} u^{\prime} e^{-i k x} d x=-\frac{1}{2 \pi k^{2}} \int_{-\pi}^{\pi} u^{\prime \prime} e^{-i k x} d x .
$$

Substituting $u^{\prime \prime}=-\lambda \epsilon u$ into (4.12) and, for convenience, using the notation $\mu=$ $\beta^{2}-1$, we have

$$
\begin{aligned}
\hat{u}_{k} & =\frac{1}{2 \pi k^{2}} \int_{-\pi}^{\pi} \lambda \epsilon u e^{-i k x} d x=\frac{\lambda}{2 \pi k^{2}}\left(\int_{-\pi}^{0} u e^{-i k x} d x+\beta^{2} \int_{0}^{\pi} u e^{-i k x} d x\right) \\
& =\frac{\lambda}{2 \pi i k^{3}}\left(\mu\left\{(-1)^{k} u(\pi)-u(0)\right\}+\int_{-\pi}^{0} u^{\prime} e^{-i k x} d x+\beta^{2} \int_{0}^{\pi} u^{\prime} e^{-i k x} d x\right) \\
& =\frac{\lambda}{2 \pi i k^{3}}\left(\mu\left[(-1)^{k} u(\pi)-u(0)\right]+\frac{\mu}{i k}\left[(-1)^{k} u^{\prime}(\pi)-u^{\prime}(0)\right]+\frac{1}{i k} \int_{-\pi}^{\pi} \lambda \epsilon u e^{-i k x} d x\right) .
\end{aligned}
$$

Therefore, the lemma is proven.

We are ready now for the next lemma.

LEMMA 4.3.

$$
\max _{v \in E 1, l} \frac{(v, v)}{(P v, P v)} \leq 1+C l N^{-3}
$$

where the constant $C$ is independent of $N$ and $l$. 
Proof. We first note that

$$
\frac{(v, v)}{(P v, P v)}=\frac{1}{1-\frac{(v, v)-(P v, P v)}{(v, v)}} .
$$

Since $v$ is in $E_{1, l}$, it can be represented by $v=\sum_{i=1}^{l} \alpha_{i} u_{i}$. We also have

$$
(P v, P v)=(v, v)-(v-P v, v)-\overline{(v-P v, v)}+(v-P v, v-P v) .
$$

Thus we get

$$
\begin{aligned}
\frac{(v, v)-(P v, P v)}{(v, v)} & \leq \frac{2|(v-P v, v)|}{(v, v)} \\
& =\frac{2 \sum_{i, j=1}^{l}\left|\alpha_{i}\right|\left|\bar{\alpha}_{j}\right|\left|\left(u_{i}-P u_{i}, u_{j}\right)\right|}{\left(\sum_{i=1}^{l}\left|\alpha_{i}\right|^{2}\right)} \\
& \leq 2 l \max _{i, j=1, \ldots, l}\left|\left(u_{i}-P u_{i}, u_{j}\right)\right| .
\end{aligned}
$$

Now we have

$$
\begin{aligned}
\left|\left(u_{i}-P u_{i}, u_{j}\right)\right|= & \left|\left(\sum_{|k|>\frac{N}{2}}\left(\hat{u_{i}}\right)_{k} e^{i k x}, \sum_{n=\infty}^{\infty}\left(\hat{u_{j}}\right)_{n} e^{i n x}\right)\right| \\
= & \left|\sum_{|k|>\frac{N}{2}} \sum_{n=\infty}^{\infty}\left(\hat{u_{i}}\right)_{k} \overline{\left(\hat{u_{j}}\right)_{n}} \int_{-\pi}^{\pi} e^{i(k-n) x} \epsilon(x) d x\right| \\
\leq & \sum_{|k|>\frac{N}{2}} \sum_{\substack{n=\infty \\
n \neq k}}^{\infty}\left|\left(\hat{u_{i}}\right)_{k}\right| \cdot\left|\overline{\left(\hat{u_{j}}\right)_{n}}\right| \cdot \frac{\left(\beta^{2}-1\right)\left|\left((-1)^{k-n}-1\right)\right|}{|k-n|} \\
& +\sum_{|k|>\frac{N}{2}} \sum_{\substack{n=\infty \\
n=k}}^{\infty}\left|\left(\hat{u_{i}}\right)_{k}\right| \cdot\left|\overline{\left(\hat{u_{j}}\right)_{n}}\right| \cdot\left(\beta^{2}+1\right) \pi .
\end{aligned}
$$

Recalling (4.11), where $\left|\hat{u}_{k}\right|$ decays like $O\left(k^{-3}\right)$ at least, we get

$$
\left|\left(u_{i}-P u_{i}, u_{j}\right)\right| \leq C N^{-3},
$$

where $C$ is a positive constant. Finally, we have

$$
\begin{aligned}
\frac{(v, v)}{(P v, P v)} & =\frac{1}{1-\frac{(v, v)-(P v, P v)}{(v, v)}} \\
& \leq 1+2 \frac{(v, v)-(P v, P v)}{(v, v)} \\
& \leq 1+C l N^{-3} .
\end{aligned}
$$

Thus the lemma is proven.

We can now state the following theory.

TheOREm 4.4. Let $\lambda_{l}^{N}$ be the Fourier-Galerkin approximation to the eigenvalue $\lambda_{l}$. Then

$$
\left|\lambda_{l}-\lambda_{l}^{N}\right| \leq C l \lambda_{l} N^{-3}
$$


where the constant $C$ depends only on the values of $u_{i}(0), u_{i}^{\prime}(0)$, and the $L_{2}$-norm of $u_{i}$ for all $i \leq l$.

Now we are ready to treat the eigenvectors. Following Strang and Fix [7, p. 234], we can state

$$
\left\|u_{l}-u_{l}^{N}\right\| \leq\left\|u_{l}-P_{N} u_{l}\right\|
$$

where $P_{N} u_{l}$ is the finite Fourier series of $u_{l}$. For the right-hand side we have the following estimate.

LEMMA 4.5.

$$
\left\|u_{l}-P_{N} u_{l}\right\| \leq C N^{-2.5}
$$

Proof. By the Parseval equality, and using Lemma 4.2, which states the Fourier coefficients of $u_{l}$ decay cubicly, we get

$$
\begin{aligned}
\left\|u_{l}-P_{N} u_{l}\right\| & =\left(\sum_{|k|>\frac{N}{2}}\left|\left(\hat{u}_{l}\right)_{k}\right|^{2}\right)^{\frac{1}{2}} \\
& \leq C\left(\sum_{|k|>\frac{N}{2}}|k|^{-6}\right)^{\frac{1}{2}} \\
& \leq C N^{-2.5} .
\end{aligned}
$$

Thus the lemma is proven.

We can therefore conclude the following theorem.

THEOREM 4.6. Let $u_{l}$ be the lth eigenfunction, and let $u_{l}^{N}$ be the solution of the Fourier-Galerkin approximation (4.2); then

$$
\left\|u_{l}-u_{l}^{N}\right\| \leq C N^{-2.5}
$$

The numerical results presented in Tables 1 and 2 conform to the theory.

5. Fourier collocation method. Let $\mathcal{I}_{\mathcal{N}}$ be the space of the trigonometric polynomial of degree $N / 2$, defined as

$$
\mathcal{I}_{\mathcal{N}}=\operatorname{span}\{(\cos (k x) \mid 0 \leq k \leq N / 2) \cup(\sin (k x) \mid 1 \leq k \leq N / 2-1)\} .
$$

For an even integer $N>0$, we consider the set of points

$$
x_{j}=-\pi+\frac{2 \pi j}{N}, \quad j=0, \ldots, N .
$$

The discrete approximations of the inner products (3.1) and (3.2) are defined by

$$
\begin{aligned}
a(u, v)_{h} & =\frac{2 \pi}{N} \sum_{j=0}^{N-1} u^{\prime}\left(x_{j}\right) \overline{v^{\prime}\left(x_{j}\right)}, \\
(u, v)_{h} & =\frac{2 \pi}{N} \sum_{j=0}^{N-1} u\left(x_{j}\right) \overline{v\left(x_{j}\right)} \epsilon\left(x_{j}\right) .
\end{aligned}
$$


Alternatively, defining $c_{j}=1,0 \neq j \neq N$, and $c_{N}=c_{0}=2$, we can redefine

$$
\begin{aligned}
a(u, v)_{h} & =\frac{2 \pi}{N} \sum_{j=0}^{N} u^{\prime}\left(x_{j}\right) \overline{v^{\prime}\left(x_{j}\right)} \frac{1}{c_{j}}, \\
(u, v)_{h} & =\frac{2 \pi}{N} \sum_{j=0}^{N} u\left(x_{j}\right) \overline{v\left(x_{j}\right)} \epsilon\left(x_{j}\right) \frac{1}{c_{j}} .
\end{aligned}
$$

Remark 5.1. Note that the bilinear form $a(u, v)_{h}$ coincides with the inner product $a(u, v)$ for trigonometrical polynomials of the right order:

$$
a(u, v)_{h}=a(u, v) \quad \text { for all } u, v \in \mathcal{I}_{\mathcal{N}} .
$$

This is a result of the exactness of the quadrature formula if $u^{\prime} v^{\prime}$ is up to degree $N-1$ [1]. One can observe that the highest degree $N$ for $u^{\prime} v^{\prime}$ is obtained when choosing $\cos \left(\frac{N}{2} x\right)$ for both $u$ and $v$. However, $\left\{\cos \left(\frac{N}{2} x\right)\right\}^{\prime}=-\frac{N}{2} \sin \left(\frac{N}{2} x\right)$ and $\sin \left(\frac{N}{2} x\right)$ vanishes at the grid points $x_{j}$ so that the quadrature formula still remains valid also for the case of highest degree $N$. Thus (5.7) is true for any $u, v$ in $\mathcal{I}_{\mathcal{N}}$.

Remark 5.2. Equation (5.6) can be rewritten as

$$
\begin{aligned}
(v, v)_{h}= & \frac{\pi}{N}\left(\left|v\left(x_{0}\right)\right|^{2} \epsilon\left(x_{0}\right)+\left|v\left(x_{\frac{N}{2}}\right)\right|^{2} \epsilon\left(x_{\frac{N}{2}}\right)\right)+\frac{2 \pi}{N} \sum_{j=1}^{\frac{N}{2}-1}\left|v\left(x_{j}\right)\right|^{2} \epsilon\left(x_{j}\right) \\
& +\frac{2 \pi}{N} \sum_{\frac{N}{2}+1}^{N-1}\left|v\left(x_{j}\right)\right|^{2} \epsilon\left(x_{j}\right)+\frac{\pi}{N}\left(\left|v\left(x_{N}\right)\right|^{2} \epsilon\left(x_{N}\right)+\left|v\left(x_{\frac{N}{2}}\right)\right|^{2} \epsilon\left(x_{\frac{N}{2}}\right)\right) .
\end{aligned}
$$

The first two terms can be identified as the trapezoidal rule [6] for $\int_{-\pi}^{0}|v(\xi)|^{2} \epsilon(\xi) d \xi$, whereas the other two terms are the same rule for $\int_{0}^{\pi}|v(\xi)|^{2} \epsilon(\xi) d \xi$. We can therefore state

$$
\left|(v, v)-(v, v)_{h}\right| \leq C N^{-2} \max \left\{\max _{-\pi \leq x<0}\left(|v|^{2} \cdot \epsilon\right)^{\prime \prime}, \max _{0 \leq x \leq \pi}\left(|v|^{2} \cdot \epsilon\right)^{\prime \prime}\right\} .
$$

5.1. Numerical scheme and its results. The collocation methods can be defined as finding $\lambda^{c}$ and $u^{c} \in \mathcal{I}_{N}$ such that

$$
a\left(u^{c}, v^{c}\right)_{h}=\lambda^{c}\left(u^{c}, v^{c}\right)_{h} \text { for all } v^{c} \in \mathcal{I}_{N} .
$$

There are several ways to realize the abstract definition of the collocation methods, and we will quote one of them: $u^{c}$ can be presented using the Lagrange trigonometrical polynomials as interpolation polynomials [4] as follows:

$$
u^{c}=\sum_{j=0}^{N-1} u^{c}\left(x_{j}\right) l_{j}(x),
$$

where

$$
l_{j}(x)=\frac{1}{N} \sin \left[N \frac{\left(x-x_{j}\right)}{2}\right] \cot \left[\frac{x-x_{j}}{2}\right]
$$


Taking $v^{c}(x)=l_{n}(x)$, we have

$$
\begin{aligned}
a(u, v)_{h} & =\frac{2 \pi}{N} \sum_{i=0}^{N-1} \sum_{j=0}^{N-1} u^{c}\left(x_{j}\right) l_{j}^{\prime}\left(x_{i}\right) l_{n}^{\prime}\left(x_{i}\right) \\
& =\frac{2 \pi}{N} \sum_{j=0}^{N-1} u^{c}\left(x_{j}\right) \sum_{i=0}^{N-1} l_{j}^{\prime}\left(x_{i}\right) l_{n}^{\prime}\left(x_{i}\right) \\
& =\frac{2 \pi}{N} \sum_{j=0}^{N-1} u^{c}\left(x_{j}\right) D_{n j}^{2},
\end{aligned}
$$

where $D^{2}=-D \cdot D$ and $\mathrm{D}$ is the first order differentiation matrix for even grid points [2], [4], [5]. Also,

$$
\begin{aligned}
(u, v)_{h} & =\frac{2 \pi}{N} \sum_{i=0}^{N-1} \sum_{j=0}^{N-1} u^{c}\left(x_{j}\right) l_{j}\left(x_{i}\right) l_{n}\left(x_{i}\right) \epsilon\left(x_{i}\right) \\
& =\frac{2 \pi}{N} \sum_{j=0}^{N-1} u^{c}\left(x_{j}\right) \sum_{i=0}^{N-1} l_{j}\left(x_{i}\right) l_{n}\left(x_{i}\right) \epsilon\left(x_{i}\right) \\
& =\frac{2 \pi}{N} \sum_{j=0}^{N-1} u^{c}\left(x_{j}\right) A_{j n},
\end{aligned}
$$

where $A=\operatorname{diag}\left\{\epsilon\left(x_{0}\right), \ldots, \epsilon\left(x_{N-1}\right)\right\}$. Then we solve the matrix equation

$$
D^{2} \mathbf{u}^{c}=\lambda^{c} A \mathbf{u}^{c}
$$

to get the approximate eigenvalues $\lambda^{c}$ and eigenfunctions $\mathbf{u}^{c}=\left[u^{c}\left(x_{0}\right), \ldots, u^{c}\left(x_{N-1}\right)\right]^{T}$.

Remark 5.3. In order to make (5.12) compatible with definition (5.6), we should replace $\epsilon\left(x_{0}\right)$ by the average $\frac{\epsilon\left(x_{0}\right)+\epsilon\left(x_{N}\right)}{2}$.

The variational formulation with odd grids can be obtained in a similar way. In Tables 3 and 4, we present the relative error for $\lambda_{l}^{c}-\lambda_{l}$. It is clear that we see second order accuracy with even grids as well as with odd grids as $N$ increases. The discrete $L_{2}$-error of $u_{l}-u_{l}^{c}$ converges with second order accuracy with even and odd grids as $N$ increases.

5.2. Error estimates for eigenvalues and eigenfunctions. Here we provide error estimates for the approximate eigenvalues and eigenfunctions of the Fourier collocation method. We first consider the approximate eigenvalues. Let $S_{l}$ be any $l$-dimensional subspace of $\mathcal{I}_{\mathcal{N}}$.

LEMMA 5.1. Let $\lambda_{l}^{c}$ be the approximation to $\lambda_{l}$ obtained by the collocation procedure, and let $S_{l}$ be any l-dimensional subspace of $\mathcal{I}_{\mathcal{N}}$. Then

$$
\lambda_{l}^{c} \leq \max _{v \in S_{l}} \frac{a(v, v)_{h}}{(v, v)_{h}} .
$$

Proof. The space $S_{l}$ is spanned by the eigenfunctions $u_{k_{1}}^{c}, \ldots, u_{k_{l}}^{c}$ for $k_{1}<\cdots<$ $k_{l}$. It follows that $k_{l} \geq l$. Now

$$
\frac{a\left(u_{k_{l}}^{c}, u_{k_{l}}^{c}\right)_{h}}{\left(u_{k_{l}}^{c}, u_{k_{l}}^{c}\right)_{h}}=\lambda_{k_{l}}^{c} \geq \lambda_{l}^{c}
$$


TABLE 3

Fourier collocation with even grids: The relative errors of eigenvalues for the case $\beta=2$ and the discrete $L_{2}$-errors of $u_{i}-u_{i}^{c}$.

\begin{tabular}{|c||c||c||c|c||c|c|}
\hline$\lambda_{i}$ & $N$ & $\lambda_{i}^{c}$ & $\frac{\left(\lambda_{i}^{c}-\lambda_{i}\right)}{\lambda_{i}}$ & Order & $\left\|u_{i}-u_{i}^{c}\right\|_{l_{2}}$ & Order \\
\hline \multirow{5}{*}{0.36987} & 16 & 0.37224 & $6.3993(-3)$ & & $4.4960(-3)$ & \\
& 32 & 0.37049 & $1.6710(-3)$ & 1.9372 & $1.1755(-3)$ & 1.9354 \\
& 64 & 0.37003 & $4.2818(-4)$ & 1.9644 & $3.0154(-4)$ & 1.9628 \\
& 128 & 0.36992 & $1.0846(-4)$ & 1.9811 & $7.6428(-5)$ & 1.9801 \\
& 256 & 0.36989 & $2.7298(-5)$ & 1.9903 & $1.9243(-5)$ & 1.9897 \\
\hline \multirow{5}{*}{0.53623} & 16 & 0.53024 & $-1.1169(-2)$ & & $7.3926(-3)$ & \\
& 32 & 0.53477 & $-2.7366(-3)$ & 2.0290 & $1.7725(-3)$ & 2.0603 \\
& 64 & 0.53587 & $-6.7697(-4)$ & 2.0152 & $4.3472(-4)$ & 2.0276 \\
& 128 & 0.53614 & $-1.6834(-4)$ & 2.0077 & $1.0771(-4)$ & 2.0130 \\
& 256 & 0.53621 & $-4.1974(-5)$ & 2.0038 & $2.6811(-5)$ & 2.0062 \\
\hline \multirow{5}{*}{1.60712} & 16 & 1.63690 & $1.8531(-2)$ & & $1.1564(-2)$ & \\
& 32 & 1.61442 & $4.5477(-3)$ & 2.0267 & $3.0650(-3)$ & 1.9157 \\
& 64 & 1.60895 & $1.1432(-3)$ & 1.9920 & $7.9696(-4)$ & 1.9433 \\
& 128 & 1.60758 & $2.8754(-4)$ & 1.9913 & $2.0372(-4)$ & 1.9679 \\
& 256 & 1.60723 & $7.2160(-5)$ & 1.9945 & $5.1533(-5)$ & 1.9830 \\
\hline \multirow{5}{*}{1.93718} & 16 & 1.89597 & $-2.1273(-2)$ & & $1.7356(-2)$ & \\
& 32 & 1.92826 & $-4.6037(-3)$ & 2.2081 & $4.8491(-3)$ & 1.8396 \\
& 64 & 1.93510 & $-1.0746(-3)$ & 2.0991 & $1.2694(-3)$ & 1.9336 \\
& 128 & 1.93668 & $-2.5988(-4)$ & 2.0478 & $3.2443(-4)$ & 1.9682 \\
& 256 & 1.93706 & $-6.3921(-5)$ & 2.0235 & $8.1999(-5)$ & 1.9842 \\
\hline \multirow{5}{*}{4.00000} & 16 & 4.00439 & $1.0977(-3)$ & & $6.8315(-2)$ & \\
& 32 & 4.00012 & $2.9270(-5)$ & 5.2288 & $1.5700(-2)$ & 2.1214 \\
& 64 & 4.00000 & $8.7981(-7)$ & 5.0561 & $3.8391(-3)$ & 2.0319 \\
& 128 & 4.00000 & $2.7229(-8)$ & 5.0140 & $9.5432(-4)$ & 2.0082 \\
& 256 & 4.00000 & $8.4864(-10)$ & 5.0039 & $2.3823(-4)$ & 2.0021 \\
\hline
\end{tabular}

TABLE 4

Fourier collocation with odd grids: The relative errors of eigenvalues for the case $\beta=2$ and the discrete $L_{2}$-errors of $u_{i}-u_{i}^{c}$.

\begin{tabular}{|c||c||c||c|c||c|c|}
\hline$\lambda_{i}$ & $N$ & $\lambda_{i}^{c}$ & $\frac{\left(\lambda_{i}^{c}-\lambda_{i}\right)}{\lambda_{i}}$ & Order & $\left\|u_{i}-u_{i}^{c}\right\|_{l_{2}}$ & Order \\
\hline & 17 & 0.37037 & $1.3299(-3)$ & & $1.0195(-2)$ & \\
0.36987 & 33 & 0.37002 & $3.8441(-4)$ & 1.7906 & $2.6389(-3)$ & 1.9499 \\
& 65 & 0.36991 & $1.0289(-4)$ & 1.9016 & $6.7366(-4)$ & 1.9698 \\
& 129 & 0.36988 & $2.6595(-5)$ & 1.9519 & $1.7035(-4)$ & 1.9835 \\
& 257 & 0.36988 & $6.7596(-6)$ & 1.9761 & $4.2842(-5)$ & 1.9914 \\
\hline & 17 & 0.53491 & $-2.4693(-3)$ & & $8.0457(-3)$ & \\
0.53623 & 33 & 0.53589 & $-6.4469(-4)$ & 1.9374 & $2.0181(-3)$ & 1.9952 \\
& 65 & 0.53615 & $-1.6437(-4)$ & 1.9717 & $5.0695(-4)$ & 1.9931 \\
& 129 & 0.53621 & $-4.1480(-5)$ & 1.9864 & $1.2715(-4)$ & 1.9953 \\
& 257 & 0.53623 & $-1.0418(-5)$ & 1.9933 & $3.1846(-5)$ & 1.9973 \\
\hline & 17 & 1.61319 & $3.7829(-3)$ & & $1.6275(-2)$ & \\
1.60712 & 33 & 1.60879 & $1.0434(-3)$ & 1.8581 & $4.5235(-3)$ & 1.8471 \\
& 65 & 1.60756 & $2.7480(-4)$ & 1.9249 & $1.2086(-3)$ & 1.9041 \\
& 129 & 1.60723 & $7.0552(-5)$ & 1.9616 & $3.1368(-4)$ & 1.9460 \\
& 257 & 1.60714 & $1.7876(-5)$ & 1.9806 & $7.9996(-5)$ & 1.9713 \\
\hline & 17 & 1.92848 & $-4.4933(-3)$ & & $3.7036(-2)$ & \\
& 33 & 1.93509 & $-1.0779(-3)$ & 2.0595 & $9.7683(-3)$ & 1.9228 \\
& 65 & 1.93667 & $-2.6181(-4)$ & 2.0416 & $2.5446(-3)$ & 1.9407 \\
& 129 & 1.93706 & $-6.4278(-5)$ & 2.0261 & $6.5220(-4)$ & 1.9641 \\
& 257 & 1.93715 & $-1.5904(-5)$ & 2.0149 & $1.6529(-4)$ & 1.9803 \\
\hline
\end{tabular}


This concludes the proof.

We are now ready to estimate $\lambda_{l}^{c}$ from above.

LEMMA 5.2.

$$
\lambda_{l}^{c} \leq \lambda_{l}\left(1+C N^{-2}\right),
$$

where $C$ is independent of $N$ (but may depend linearly on l).

Proof. Let $J_{N}(=J)$ be the orthogonal projection (in the usual $L^{2}$ sense) of $H^{2}$ to $\mathcal{I}_{\mathcal{N}}$. Let $S_{l}=J E_{1, l}=\operatorname{span}\left\{J u_{1}, \ldots, J u_{l}\right\}$ in Lemma 5.1 to get

$$
\lambda_{l}^{c} \leq \max _{v \in J E_{1, l}} \frac{a(v, v)_{h}}{(v, v)_{h}}=\max _{v \in E_{1, l}} \frac{a(J v, J v)_{h}}{(J v, J v)_{h}} .
$$

Then

$$
\lambda_{l}^{c} \leq \max _{v \in E_{1, l}} \frac{a(v, v)}{(v, v)} \cdot \frac{a(J v, J v)_{h}}{a(v, v)} \cdot \frac{(v, v)}{(J v, J v)} \cdot \frac{(J v, J v)}{(J v, J v)_{h}} .
$$

From Lemma 3.2, we have

$$
\max _{v \in E_{1, l}} \frac{a(v, v)}{(v, v)}=\lambda_{l}
$$

Also from the exactness of the trapezoidal rule and the fact that $J$ is an orthogonal projection, it is true that

$$
a(J v, J v)_{h}=a(J v, J v) \leq a(v, v) .
$$

Due to Lemma 4.3 (with the same proof for $J$ replacing $P$ ), we have

$$
\max _{v \in E_{1, l}} \frac{(v, v)}{(J v, J v)} \leq 1+C l N^{-3}
$$

Also, Remark 5.2 gives

$$
\frac{(J v, J v)}{(J v, J v)_{h}} \leq 1+C N^{-2}
$$

and so the lemma is proven.

We will now try to get a lower bound for $\lambda_{l}^{c}$. Define $E_{1, l}^{c}=\operatorname{span}\left\{u_{1}^{c}, \ldots, u_{l}^{c}\right\}$. From the minmax theorem, we have

$$
\lambda_{l} \leq \max _{v \in E_{1, l}^{c}} \frac{a(v, v)}{(v, v)} .
$$

It is also clear that

$$
\lambda_{l}^{c}=\max _{v \in E_{1, l}^{c}} \frac{a(v, v)_{h}}{(v, v)_{h}} .
$$

We can now state the following lemma.

LEMMA 5.3.

$$
\lambda_{l} \leq \lambda_{l}^{c}\left(1+C N^{-2}\right),
$$


where $C$ is independent of $N$ (but may depend linearly on l).

Proof. We start from

$$
\begin{aligned}
\lambda_{l} & \leq \max _{v \in E_{1, l}^{c}} \frac{a(v, v)}{(v, v)} \\
& =\max _{v \in E_{1, l}^{c}} \frac{a(v, v)_{h}}{(v, v)_{h}} \cdot \frac{a(v, v)}{a(v, v)_{h}} \cdot \frac{(v, v)_{h}}{(v, v)} .
\end{aligned}
$$

Since $v \in \mathcal{I}_{\mathcal{N}}$, we have $a(v, v)=a(v, v)_{h}$. Also, because of the trapezoidal rule estimate

$$
\left|(v, v)_{h}-(v, v)\right| \leq C N^{-2},
$$

and therefore the lemma is proven.

We can now conclude the following theorem.

THEOREM 5.4. Let $\lambda_{l}^{c}$ be the approximation to $\lambda_{l}$ obtained by the collocation procedure. Then

$$
\left|\lambda_{l}^{c}-\lambda_{l}\right| \leq C \lambda_{l} N^{-2},
$$

where $C$ is independent of $N$.

We now turn to the eigenfunctions. The set $u_{1}^{c}, u_{2}^{c}, \ldots, u_{N}^{c}$ forms an orthogonal basis for $\mathcal{I}_{\mathcal{N}}$. Then we can express the orthogonal projection $J u_{l}$ of $u_{l}$ into the subspace $\mathcal{I}_{\mathcal{N}}$ as the following:

$$
J u_{l}=\sum_{j=1}^{N}\left(J u_{l}, u_{j}^{c}\right)_{h} u_{j}^{c} .
$$

By subtracting the following variational formulations,

$$
\begin{aligned}
\lambda_{l}\left(u_{l}, u_{j}^{c}\right) & =a\left(u_{l}, u_{j}^{c}\right), \\
\lambda_{j}^{c}\left(J u_{l}, u_{j}^{c}\right)_{h} & =a\left(J u_{l}, u_{j}^{c}\right)_{h}=a\left(J u_{l}, u_{j}^{c}\right),
\end{aligned}
$$

we have

$$
\begin{aligned}
\left(\lambda_{j}^{c}-\lambda_{l}\right)\left(J u_{l}, u_{j}^{c}\right)_{h} & =a\left(J u_{l}, u_{j}^{c}\right)-a\left(u_{l}, u_{j}^{c}\right)-\lambda_{l}\left[\left(J u_{l}, u_{j}^{c}\right)_{h}-\left(u_{l}, u_{j}^{c}\right)\right] \\
& =-a\left(u_{l}-J u_{l}, u_{j}^{c}\right)+\lambda_{l}\left[\left(u_{l}, u_{j}^{c}\right)-\left(J u_{l}, u_{j}^{c}\right)_{h}\right] .
\end{aligned}
$$

Since $a\left(u_{l}-J u_{l}, u_{j}^{c}\right)=0$, we have

$$
\begin{aligned}
\left|\left(J u_{l}, u_{j}^{c}\right)_{h}\right| & \leq \frac{\lambda_{l}}{\left|\lambda_{j}^{c}-\lambda_{l}\right|} \cdot\left|\left(u_{l}, u_{j}^{c}\right)-\left(J u_{l}, u_{j}^{c}\right)_{h}\right| \\
& \leq \frac{\lambda_{l}}{\left|\lambda_{j}^{c}-\lambda_{l}\right|} \cdot\left\{\left|\left(u_{l}, u_{j}^{c}\right)-\left(u_{l}, u_{j}^{c}\right)_{h}\right|+\left|\left(u_{l}, u_{j}^{c}\right)_{h}-\left(J u_{l}, u_{j}^{c}\right)_{h}\right|\right\} .
\end{aligned}
$$

From the Schwarz inequality and $\left(u_{j}^{c}, u_{j}^{c}\right)_{h}=1$, we have

$$
\begin{aligned}
\left|\left(u_{l}-J u_{l}, u_{j}^{c}\right)_{h}\right| & \leq\left\|u_{l}-J u_{l}\right\|_{h} \cdot\left\|u_{j}^{c}\right\|_{h} \\
& \leq C N^{-2}
\end{aligned}
$$


where $\|u\|_{h}=\sqrt{(u, u)_{h}}$. Using the trapezoidal rule as in (5.8), we have

$$
\left|\left(u_{l}, u_{j}^{c}\right)-\left(u_{l}, u_{j}^{c}\right)_{h}\right| \leq C N^{-2}
$$

Then, following [7],

$$
\left\|J u_{l}-\beta u_{l}^{c}\right\|_{h}=\sqrt{\sum_{\substack{j=1 \\ j \neq l}}^{N}\left|\left(J u_{l}, u_{j}^{c}\right)_{h}\right|^{2}} \leq \rho C N^{-2},
$$

where $\beta=\left(J u_{l}, u_{l}^{c}\right)_{h}$ and $\rho$ is a separation constant for the eigenvalues as in $[7$, pp. 234-235]. From (5.8) and $\left\|u_{l}\right\|=\left\|u_{l}^{c}\right\|_{h}=1$,

$$
\left\|u_{l}\right\|_{h} \leq\left\|u_{l}\right\|+C N^{-2}=\left\|u_{l}^{c}\right\|_{h}+C N^{-2} .
$$

Then, following [7], we have

$$
\left\|u_{l}-u_{l}^{c}\right\|_{h} \leq\left\|u_{l}-J u_{l}\right\|_{h}+\left\|J u_{l}-\beta u_{l}^{c}\right\|_{h}+\left\|\beta u_{l}^{c}-u_{l}^{c}\right\|_{h} \leq C N^{-2} .
$$

We can therefore conclude the following theorem.

THEOREM 5.5. Let $u_{l}$ be the lth eigenfunction, and let $u_{l}^{c}$ be the solution of the Fourier collocation approximation (5.9); then

$$
\left\|u_{l}-u_{l}^{c}\right\|_{h} \leq C N^{-2} \text {. }
$$

Due to the equivalence of the norms in finite space, the discrete $L_{2}$-error of $u_{l}-u_{l}^{c}$ also converges with $O\left(N^{-2}\right)$.

6. Accuracy enhancement for the collocation method. A simple trick can be used in order to enhance the accuracy of the Fourier collocation method. We expand the discontinuous coefficient function $\epsilon(x)$ in the finite Fourier series represented by

$$
\epsilon^{N}(x)=\sum_{k=-\frac{N}{2}}^{\frac{N}{2}}\left(\hat{\epsilon}^{N}\right)_{k} e^{i k x},
$$

where the Fourier coefficients are defined as

$$
\left(\hat{\epsilon}^{N}\right)_{k}=\frac{1}{2 \pi} \int_{-\pi}^{\pi} \epsilon(x) e^{-i k x} d x .
$$

Now, instead of (5.4), defining

$$
(u, v)_{h}=\frac{2 \pi}{N} \sum_{j=0}^{N-1} u\left(x_{j}\right) \overline{v\left(x_{j}\right)} \epsilon^{N}\left(x_{j}\right)
$$

in the variational formulation (5.9), we have the scheme as follows:

$$
D^{2} \mathbf{u}^{c}=\lambda^{c} A \mathbf{u}^{c}
$$

where $A=\operatorname{diag}\left\{\epsilon^{N}\left(x_{0}\right), \ldots, \epsilon^{N}\left(x_{N-1}\right)\right\}$ and $D^{2}$ is the same as defined in (5.12).

The numerical results are presented in the Tables 5 and 6 for even and odd grids, respectively. The accuracy is now the same accuracy as for the Galerkin method! An analysis for this will appear in a future paper. 
TABLE 5

The accuracy enhancement for collocation with even grids: The relative errors of eigenvalues for the case $\beta=2$ and the discrete $L_{2}$-errors of $u_{i}-u_{i}^{c}$ using $\epsilon^{N}(x)$.

\begin{tabular}{|c||c||c||c|c||c|c|}
\hline$\lambda_{i}$ & $N$ & $\lambda_{i}^{c}$ & $\frac{\left(\lambda_{i}^{c}-\lambda_{i}\right)}{\lambda_{i}}$ & Order & $\left\|u_{i}-u_{i}^{c}\right\|_{l_{2}}$ & Order \\
\hline & 16 & 0.36995 & $2.0453(-4)$ & & $5.1956(-4)$ & \\
0.36987 & 32 & 0.36988 & $2.1664(-5)$ & 3.2389 & $1.0071(-4)$ & 2.3671 \\
& 64 & 0.36988 & $2.4904(-6)$ & 3.1208 & $1.9120(-5)$ & 2.3970 \\
& 128 & 0.36988 & $2.9842(-7)$ & 3.0609 & $3.5138(-6)$ & 2.4440 \\
& 256 & 0.36987 & $3.6546(-8)$ & 3.0296 & $6.3363(-7)$ & 2.4713 \\
\hline & 16 & 0.53624 & $7.7676(-6)$ & & $9.7460(-4)$ & \\
0.53623 & 32 & 0.53624 & $5.7076(-6)$ & 0.4445 & $1.4544(-4)$ & 2.7444 \\
& 64 & 0.53623 & $1.0179(-6)$ & 2.4873 & $2.2753(-5)$ & 2.6764 \\
& 128 & 0.53623 & $1.4643(-7)$ & 2.7973 & $3.7110(-6)$ & 2.6161 \\
& 256 & 0.53623 & $1.9435(-8)$ & 2.9135 & $6.2557(-7)$ & 2.5686 \\
\hline \multirow{5}{*}{1.60712} & 16 & 1.60674 & $-2.3345(-4)$ & & $4.9371(-3)$ & \\
& 32 & 1.60714 & $1.5379(-5)$ & 3.9241 & $4.7815(-4)$ & 3.3681 \\
& 64 & 1.60712 & $3.2157(-6)$ & 2.2577 & $6.7468(-5)$ & 2.8250 \\
& 128 & 1.60712 & $4.5610(-7)$ & 2.8177 & $1.0873(-5)$ & 2.6334 \\
& 256 & 1.60712 & $5.9832(-8)$ & 2.9304 & $1.8450(-6)$ & 2.5591 \\
\hline \multirow{5}{*}{1.93718} & 16 & 1.94119 & $2.0698(-3)$ & & $6.8043(-3)$ & \\
& 32 & 1.93744 & $1.3393(-4)$ & 3.9500 & $6.3418(-4)$ & 3.4235 \\
& 64 & 1.93721 & $1.3685(-5)$ & 3.2908 & $1.0359(-4)$ & 2.6140 \\
& 128 & 1.93718 & $1.5887(-6)$ & 3.1067 & $1.8498(-5)$ & 2.4855 \\
& 256 & 1.93718 & $1.9292(-7)$ & 3.0418 & $3.3183(-6)$ & 2.4789 \\
\hline & 16 & 3.92531 & $-1.8673(-2)$ & & $3.9832(-2)$ & \\
& 32 & 3.99878 & $-3.0425(-4)$ & 5.9395 & $1.8962(-3)$ & 4.3927 \\
4.00000 & 64 & 3.99995 & $-1.3559(-5)$ & 4.4880 & $2.0989(-4)$ & 3.1754 \\
& 128 & 4.00000 & $-7.3809(-7)$ & 4.1993 & $2.5516(-5)$ & 3.0402 \\
& 256 & 4.00000 & $-4.3296(-8)$ & 4.0915 & $3.1659(-6)$ & 3.0107 \\
\hline
\end{tabular}

TABLE 6

The accuracy enhancement for collocation with odd grids: The relative errors of eigenvalues for the case $\beta=2$ and the discrete $L_{2}$-errors of $u_{i}-u_{i}^{c}$ using $\epsilon^{N}(x)$.

\begin{tabular}{|r||c||c||c|c||c|c|}
\hline$\lambda_{i}$ & $N$ & $\lambda_{i}^{c}$ & $\frac{\left(\lambda_{i}^{c}-\lambda_{i}\right)}{\lambda_{i}}$ & Order & $\left\|u_{i}-u_{i}^{c}\right\|_{l_{2}}$ & Order \\
\hline & 17 & 0.36994 & $1.7576(-4)$ & & $5.8880(-4)$ & \\
& 33 & 0.36988 & $1.9991(-5)$ & 3.1362 & $1.0629(-4)$ & 2.4698 \\
0.36987 & 65 & 0.36988 & $2.3892(-6)$ & 3.0647 & $1.9258(-5)$ & 2.4644 \\
& 129 & 0.36988 & $2.9220(-7)$ & 3.0315 & $3.4570(-6)$ & 2.4778 \\
& 257 & 0.36987 & $3.6169(-8)$ & 3.0141 & $6.1624(-7)$ & 2.4880 \\
\hline \multirow{5}{*}{0.53623} & 17 & 0.53626 & $4.4494(-5)$ & & $8.1441(-4)$ & \\
& 33 & 0.53624 & $8.1111(-6)$ & 2.4556 & $1.2636(-4)$ & 2.6882 \\
& 65 & 0.53623 & $1.1708(-6)$ & 2.7924 & $2.0471(-5)$ & 2.6259 \\
& 129 & 0.53623 & $1.5604(-7)$ & 2.9075 & $3.4310(-6)$ & 2.5768 \\
& 257 & 0.53623 & $2.0042(-8)$ & 2.9608 & $5.8871(-7)$ & 2.5430 \\
\hline \multirow{5}{*}{1.60712} & 17 & 1.60723 & $7.1745(-5)$ & & $3.5514(-3)$ & \\
& 33 & 1.60716 & $2.4805(-5)$ & 1.5323 & $3.9880(-4)$ & 3.1547 \\
& 65 & 1.60712 & $3.6264(-6)$ & 2.7740 & $6.0783(-5)$ & 2.7139 \\
& 129 & 1.60712 & $4.7742(-7)$ & 2.9252 & $1.0143(-5)$ & 2.5832 \\
& 257 & 1.60712 & $6.1050(-8)$ & 2.9672 & $1.7484(-6)$ & 2.5364 \\
\hline \multirow{5}{*}{1.93718} & 17 & 1.93983 & $1.3694(-3)$ & & $5.1304(-3)$ & \\
& 33 & 1.93740 & $1.1435(-4)$ & 3.5820 & $6.1267(-4)$ & 3.0659 \\
& 65 & 1.93721 & $1.2826(-5)$ & 3.1563 & $1.0292(-4)$ & 2.5736 \\
& 129 & 1.93718 & $1.5433(-6)$ & 3.0550 & $1.8186(-5)$ & 2.5006 \\
& 257 & 1.93718 & $1.9029(-7)$ & 3.0197 & $3.2305(-6)$ & 2.4930 \\
\hline
\end{tabular}


Acknowledgments. The authors would like to thank Bertil Gustafsson, Seymour Parter, and Wai-Sun Don for several useful suggestions and discussions regarding this paper.

\section{REFERENCES}

[1] C. Canuto, M. Y. Hussaini, A. Quarteroni, and T. A. Zang, Spectral Methods in Fluid Dynamics, Springer Ser. Comput. Phys., Springer-Verlag, New York, 1988.

[2] D. Funaro, Polynomial Approximation of Differential Equations, Springer-Verlag, New York, 1991.

[3] G. H. Golub and C. Van Loan, Matrix Computations, The Johns Hopkins University Press, Baltimore, MD, 1996.

[4] D. Gottlieb, M. Y. Hussaini, And S. A. Orszag, Theory and Applications of Spectral Methods, in Spectral Methods for Partial Differential Equations, R. Voigt, D. Gottlieb, and M.Y. Hussaini, eds., SIAM, Philadelphia, 1984, pp. 1-54.

[5] D. Gottlieb and S. A. Orszag, Numerical Analysis of Spectral Methods: Theory and Application, CMBS-NSF Regional Conf. Ser. Appl. Math. 26, SIAM, Philadelphia, 1977.

[6] E. Isaacson and H. B. Keller, Analysis of Numerical Methods, John Wiley \& Sons, New York, 1966.

[7] G. Strang and G. Fix, An Analysis of The Finite Element Method, Prentice-Hall, Englewood Cliffs, NJ, 1973. 